\title{
\WE ARE FROM THE MITTELSTAND, YOU KNOW.« ESSAYISTISCHE ANMERKUNGEN ZUR VERORTUNG DER DEUTSCHEN POPMUSIK
}

\author{
Barbara Hornberger \\ »Nun, da sich der Vorhang der Nacht von der Bühne hebt, \\ kann das Spiel beginnen, \\ das uns vom Drama einer Kultur berichtet. «1
}

Ein Hauch von Vergangenheit scheint diese Zeilen zu umwehen. In pathetisch-lyrischem Ton wird ein Drama angekündigt, ein Drama, das eine ganze Kultur erfasst. Historische Katastrophen wie der Untergang von Troja oder Pompeji, verlorene Kulturen wie die Hochkultur Ägypten kommen einem in den Sinn. Bedeutungsschwanger und unheilverkündend wirkt der Satz, wie aus der Zeit gefallen.

Eine Mischung aus hohem Ton und Untergangsszenario, die nicht von ungefähr an die bürgerliche Kultur des 18. und 19. Jahrhunderts erinnert. Denn es ist das deutsche Bürgertum, das die Antike als Inspiration, mehr noch: als Vorlage für die eigene Kultur und Identität, wiederentdeckt. »Der einzige Weg für uns, groß, ja, wenn es möglich ist, unnachahmlich zu werden, ist die Nachahmung der Alten « (Winckelmann 1995: 4). ${ }^{2}$ Sie, die Griechen, haben den guten Geschmack in die Welt gebracht, an ihnen orientiert sich das bürgerliche Ideal. »Dem Wahren, Schoenen, Guten« heißt es, abgeleitet von Platon, programmatisch über dem Eingangsportal der 1880 eröffneten Frankfurter Alten Oper, variiert als "dem Wahren, Guten, Schoenen" ist es Teil der Jugendstil-Fassade des Theaters Lübeck (erbaut 1908). Das ist kein Zufall: Das Theater, insbesondere das bürgerliche Trauerspiel, hat sich im 19. Jahrhundert als moralische Anstalt und Ort bürgerlicher Selbstverständigung etabliert. Auch darum klingt das oben Zitierte nach dem 19. Jahrhundert - Vorhang und Drama sind die Insignien des Bildungstempels

1 Die Fantastischen Vier: $\gg$ MfG « (1999).

2 Zuerst 1755. 
Theater, in dem die »Dramen der Kultur « zu Belehrung und Warnung vorgetragen werden. Zugleich wird mit dem »Drama einer Kultur « eine bürgerlich-kulturpessimistische Perspektive eröffnet, die sich im 19. Jahrhundert als Gegenbewegung zum Fortschrittsglauben entwickelt und kulturell Besitzstandswahrung betreibt, indem sie angesichts der Modernisierung Untergangsszenarien beschwört.

Dies alles hätte mit populärer Kultur nur sehr entfernt zu tun, tauchte dieses Zitat nicht gleich zweimal an prominenter und populärer Stelle auf: Erstens in dem Bond-Film The Spy Who Loved Me (1977). Dort ist der Text »im Original « zu hören, nämlich als deutschsprachiger (bzw. im Original als englischsprachiger) Teil der Sound and Light Show an den Pyramiden von Gizeh. ${ }^{3}$ Hier fungiert der Text als Teil der für die Bond-Reihe obligatorischen exotischen Kulisse, die den Ort für eine erste Auseinandersetzung zwischen Bond und dem »Beißer« darstellt. Als solche ist sie diegetisch motiviert und plausibel. Zweitens im Jahr 1999 als Eingangszeilen zum Song »MfG « der Fantastischen Vier. Während der Text im Bond-Film als Teil der exotischen Kulisse passend wirkt, ist er als Intro eines HipHop-Songs durchaus irritierend: Ist es doch aus konservativer Sicht lange das Populäre gewesen, das eben jenes »Drama einer Kultur « bedeutete, das die Fantastischen Vier nun ihrerseits in einem Reigen von Abkürzungen beschwören. Aus kulturpessimistischer Perspektive treiben sie also den Teufel mit dem Beelzebub aus. Verfechter der Durchmischungs-These hingegen könnten das Zitat dieser aus dem Kontext der Bildungsreisen entlehnten Zeilen in einem HipHop-Song als Beleg dafür nehmen, dass U- und E-Kultur längst in Harmonie vereint, die Dichotomie des 19. Jahrhunderts in einem fröhlichen »Anything goes« aufgelöst sei.

Viel interessanter als eine erneute Diskussion der Vermischungs- oder Dichotomiefrage ist aber, dass die Übernahme des bildungsbürgerlich konnotierten Texts ins Populäre ein Verhältnis aufscheinen lässt, das sonst selten in den Blick gerät: Das Verhältnis nämlich, das die deutsche Popmusik sowohl seitens ihrer Künstler als auch seitens ihrer Kritiker und Theoretiker - zur bürgerlichen Kultur hat, zu der Kultur also, die als Leitkultur und zugleich Feindbild die Debatte um das Populäre immer schon mitbestimmt. Dieses Verhältnis ist wechselhaft und kompliziert, es bestimmt die Perspektive auf das Populäre, insbesondere da, wo ästhetische und moralische Wertungen ins Spiel kommen. Inwiefern solche Wertungen, z.B. seitens der

3 Zur Show siehe http://www.youtube.com/watch?v=nTCYSbBhWOw (Zugriff: 3.7.2014). Die Show wird in acht Sprachen gezeigt: Arabisch, Deutsch, Englisch, Französisch, Italienisch, Japanisch, Russisch, Spanisch. Zum Bond-Film: http:// www. youtube.com/watch?v=AxNyEUMewzg (Zugriff: 3.7.2014). 
Kritik, geprägt sind von einer dem bürgerlichen Bildungsideal folgenden Agenda, zeigt sich insbesondere da, wo Transferprozesse stattfinden: vom US-amerikanischen oder britischen Raum in den deutschen, vom Underground in den Mainstream. Wenn der Rock'n'Roll zur Teenagermusik wird, der Punk zur Neuen Deutschen Welle oder der HipHop zum Deutschrap, wird die Frage nach Authentizität und Affirmation, Entfremdung und Ausverkauf diskutiert - ohne dass deutlich wird, welche bürgerlichen Wertungen hinter den Argumenten verborgen sind. Die soziale und ideologische Verortung vieler Musiker, insbesondere aber Kritiker und Forscher, im Mittelstand ist in diesen Debatten häufig ein blinder Fleck: Sie wird nicht selbstreflexiv markiert, sondern verschämt ignoriert. Damit gerät ihre Sehnsucht nach dem Anderen, Widerständigen zum heimlichen Wertungsmaßstab des Populären - was zu Abwertungen all dessen führt, was diesem Maßstab nicht folgen mag.

\section{Die populäre und die bürgerliche Kultur}

Das Verhältnis des Populären zum Bürgerlichen ist historisch und ideologisch ein Verhältnis der Distanz: Populärkultur gilt auf der einen Seite »als Gegensatz, Abfall oder Widersacher der hohen Kultur, als Inbegriff gefährlichen Schunds oder, im besseren Fall, herabgesunkenen, trivialisierten Kulturguts« (Hecken 2007: 7). Scheinbar ohne ästhetischen Wert und mit zweifelhafter (moralischer) Herkunft kann sie von Seiten der Bildungsbeflissenen nur bekämpft, bestenfalls als »kultureller Übergangswert (Ackerknecht 2007: $143)^{4}$ vorübergehend geduldet werden. Auf der anderen Seite wird die populäre Kultur, seit den 1980er Jahren verstärkt auch im deutschsprachigen Raum, begriffen als Kultur von unten, als etwas, das rebellisch ist, aus dem Geist des Widerstands entsteht, formuliert von Arbeiterjugendlichen, Schwarzen oder Latinos. In diesem Sinne jedenfalls argumentieren wichtige Theorien zu populärer Kultur. Einflussreiche Schriften - etwa Hebdiges Subculture. The Meaning of Style (1979) oder Willis' Profane Culture (1978) zeigen z.B. die soziale und kulturelle Verortung der britischen Popmusikstile in der Arbeiterklasse. Auch die US-amerikanischen Cultural Studies betonen das Widerstandspotential der populären Kultur, ihre anti-elitäre Grundierung und ihre Verarbeitung von Unterdrückung aufgrund von Race, Class und Gender. Diese Entdeckung des Populären als Ort des Widerständigen ist auch begleitet von avantgardistischen Konzepten der Kunst, die das Spiel

4 Zuerst 1934. 
mit der Oberfläche und mit dem Seriellen aufgreifen. ${ }^{5}$ Insofern schließt die nobilitierte Auffassung des Populären als Widerstand an einen sich verändernden Kunstbegriff an. Gemeinsam ist den Positionen, dass das Populäre zunächst einmal nicht als genuiner Teil bürgerlicher (Hoch-)Kultur verstanden wird, sondern als etwas, was neben ihr steht und wahlweise als billiger und standardisierter Abklatsch von Kunst abgelehnt oder als Selbstausdruck der Unterprivilegierten, der »subordinated« oder »disempowered people« (Fiske 1989: 1), bewundert werden kann. Speziell die dem Populären gegenüber aufgeschlossene, ja, zugewandte Position der Cultural Studies hat sich inzwischen zu einer Mainstream-Position entwickelt. Allerdings zu einer Mainstream-Position, die ihrerseits den Mainstream oft ablehnt. Das Populäre wird vor allem dann geschätzt, wenn es eben nicht in einem zählbaren Sinne populär ist, wenn ihm stattdessen ein »prägende[r], differentielle[r] Charakter« (Hecken 1997: 8) zugesprochen werden kann.

In der Aneignung der in der Tat Augen öffnenden und Perspektiven erweiternden Schriften der Cultural Studies durch die deutsche Popmusikforschung geriet allerdings aus dem Blick, dass die deutsche populäre Musik in den meisten Fällen gerade nicht an den Rändern oder in der unteren Schicht der Gesellschaft entsteht. Ihre Protagonisten entstammen häufig dem Mittelstand, wie Thomas D. auf MTV erklärte (vgl. Jürgens 1999; Wagner 1999: 235). Sie ist außerdem eng verwoben mit den stilistischen Entwicklungen der britischen und US-amerikanischen Musik, die importiert und imitiert, aber auch integriert und angeeignet wird. Gerade diese deutsche Aneignung importierter Kultur wird aber, insbesondere wenn sie erfolgreich ist, häufig als Verfälschung, Verflachung und Enteignung wahrgenommen: Peter Kraus killt den Rock'n'Roll, Drafi Deutscher versklavt den Beat und die Fantastischen Vier beklauen den von den Migranten für sich beanspruchten HipHop. Die deutschen Künstler und Produzenten erscheinen in diesem Szenario als skrupellose Kulturgangster, die das Rebellische, Authentische der US-amerikanischen und britischen Stile ausbeuten, bis nichts mehr davon übrig ist.

Nun lassen sich auf der einen Seite interkulturelle Transferprozesse natürlich immer als kulturelle Verlustgeschichten schreiben. Vor dem Hintergrund einer sozial und geografisch festzumachenden >Originalität des Kunstwerks < erscheinen viele der deutschen Versionen wie >Fälschungen <. Auf der anderen Seite gilt: In einem globalen Markt ist auch die Zirkulation kultureller Waren potentiell global und diese Zirkulation ist nicht folgenlos. Die Erwartung, populäre Musikkulturen sollten, einmal als subkulturell ausgeformt und anerkannt, gefälligst so bleiben wie sie sind, ist eine, die Kultur nicht

5 Am bekanntesten sicher: Andy Warhols Siebdruck-Serien und die arbeitsteilige, kollektive Arbeitsweise in der Factory. 
als Prozess, sondern als Museum versteht. Ich möchte stattdessen eine Lesart dieser Transferprozesse favorisieren, die die Integration, Aneignung und Anverwandlung importierter Stile nicht in erster Linie als Ausbeutung wahrnimmt, sondern als Prozess einer produktiven kulturellen Veränderung, der nicht nur die importierte Musik, sondern auch die Importkultur betrifft, in einigen Fällen sogar zurückwirkt auf die internationale Zirkulation von Kultur. Der Prozess der Aneignung erfolgt in einem Wechselspiel aus der importierten Kultur und der eigenen, die ich hier - provisorisch - als eine bürgerliche bezeichne.

Provisorisch deshalb, weil der Begriff des Bürgerlichen nur schwer zu definieren ist. Historisch situiert im 18. und 19. Jahrhundert, ist die Epoche des Bürgertums mit dem 1. Weltkrieg eigentlich beendet, aber wenn man nach der sozial und kulturell dominierenden Kraft, nach der gesellschaftlichen Mitte in Deutschland fragt, wird man immer wieder auf das Erbe dieses Bürgertums stoßen, das heute eher als Mittelschicht oder Mittelstand gefasst wird. Die damit verbundene Kultur im Sinne von Selbstverständigung, Identität und Distinktion trägt an vielen Stellen das Erbe des 19. Jahrhunderts in sich, ist aber auch Träger der Ideen und Umwälzungen, die sich im 20. Jahrhundert politisch, gesellschaftlich und ästhetisch durchgesetzt haben. Das Bürgerliche ist, insbesondere seit den 1950er Jahren, keine stabile Größe, sondern befindet sich in einem dauernden Prozess der kulturellen und politischen Transformation und ist daher ausgesprochen heterogen.

»Je strenger man die sozio-ökonomischen Kriterien fasste, desto uneinheitlicher und leerer blieb das Kulturmuster der betreffenden Gruppe. Je mehr man aber von kulturellen Gemeinsamkeiten ausging, desto bunter erwies sich die soziale Zusammensetzung. Im ganzen hat die Forschung unsere Vorstellung vom >Bürgertum`als einem homogenen Träger der bürgerlichen Kultur nicht konsolidiert, sondern problematisiert« (Tenbruck 1986: 263).

Zum Bürgertum gehören Studienräte ebenso wie Facharbeiter oder Vertreter der Kreativ-, Kultur- und Sozialberufe. Es trägt daher ebenso konservative wie auch z.B. postmaterialistische Züge. Von einer bürgerlichen Kultur kann dennoch gesprochen werden, und zwar dort, wo sie repräsentativ, meinungsprägend, hegemonial wirkt. Dazu gehört die Presse ebenso wie die Wissenschaft, hier wird bürgerliche Kultur zwar häufig kritisiert, aber auch reproduziert:

»Bei der Genese der historischen Geisteswissenschaften (Philologien, aber auch Theater- und Musikwissenschaft) ist es unmittelbar einsichtig, dass sie ebenso der Analyse wie der Propaganda bürgerlicher Kultur dienten. Vor 
allem die Geisteswissenschaften setzen und legitimierten den Geltungsanspruch bürgerlicher Kultur« (Albrecht 2002: 27).

Für das Verhältnis der aus der bürgerlichen Mittelschicht kommenden Vertreter von Kritik und Forschung zur populären Musik spielen vor allem zwei Dispositionen der populären Musik eine Rolle, die gleichermaßen Distinktion versprechen: ästhetische Komplexität und Subversion. So unterschiedlich, ja, widersprüchlich, diese auch sind, beide sind auf sehr unterschiedliche Art und Weise in der Lage, die populäre Musik als künstlerisch oder ideologisch wertvoll zu adeln, indem sie an Traditionslinien und Selbstverständnisse bürgerlicher Kultur anknüpfen. Sie markieren ein konservatives und ein progressives Feld, und beide Felder sind letztlich geprägt von einer Bildungselite und ihren ästhetischen und ideologischen Ansprüchen.

\section{Komplexität}

Die Wertschätzung von ästhetischer Komplexität ist ein Erbe des 18. und 19. Jahrhunderts. Sie fordert Aufmerksamkeit und verspricht Erbauung. Tony Bennet (2010) beschreibt den bürgerlichen Blick als diszipliniert, vernünftig, aufmerksam und gebildet und stellt inn einem ungebildeten, gaffenden, betäubten (populären) Blick gegenüber. Analog dazu kann auch das bürgerliche Hören verstanden werden als eines, das bereit und in der Lage ist, Disziplin, Wissen und Aufmerksamkeit zu investieren und sich eine komplexe Struktur anzueignen. Wie sich das Museum als Ort des bürgerlichen Blicks zum Fernsehen verhält, verhält sich im Feld der populären Musik das CanKonzert zum Ballermann: Das eine gilt als künstlerisch wertvoll und bildend, das andere als kommerziell, banal und betäubend. Ein solches Regime des anspruchsvollen Hörens ist eng verknüpft mit den Institutionen, in denen die bürgerliche Mittelschicht und ihr Verständnis von Kunst dominiert: Schule und Universität, Teile des Feuilletons und der Kulturförderung. Hier wird häufig auf das Tradierte, Bewährte, Kanonisierte gesetzt. Die Platzierung der Beatles im niedersächsischen Zentralabitur 2015 als einziges Thema im Bereich Popmusik im Zeitraum 2006-2016 folgt ganz dieser Logik. ${ }^{6}$

6 Vgl. NiBiS: http://www.nibis.de/nibis.php?menid=3617 (Zugriff: 3.7.2014). Interessant ist die Begründung: »Dieser Schwerpunkt ermöglicht den Schülerinnen und Schülern exemplarische Erfahrungen mit der Musik einer Band, die wie kaum eine andere die Rock- und Pop-Kultur bis heute geprägt und an Beliebtheit und Präsenz kaum eingebüßt hat « (Niedersächsisches Kultusministerium, Themen des Zentralabiturs, Juli 2012). Man fragt sich, für welche Erfahrungen die Beatles "exemplarisch" stehen sollen - Erfahrungen mit populärer Musik dürften die Schüler im Jahr 2015 auch so haben. Und dass die Beatles an 
Eine Figur, die im 18. und 19. Jahrhundert ästhetische Komplexität zugleich präsentiert und vereinfacht und damit zwischen der Kunst und dem Populären steht, ist der Virtuose. Er ist jemand, der etwas sehr gut kann und dieses Können zur Geltung bringt. Der Virtuose »muss nicht nur musikalisch, sondern auch performativ überzeugen« (Hügel 2003: 491). Sein herausragendes professionelles Können braucht als Gegenüber einen Kenner, der es schätzen kann. Zugleich entlastet der Virtuose den Zuhörer, der nun auch Zuschauer ist, weil er ihm etwas weniger Konzentration auf das Hören abfordert und ihm optisch etwas bietet. „Das musikalisch Dargebotene ist eben mehr als Musik, es ist eine Präsentation vom Ich des Musikers. Selbstdarstellung und Rollendarstellung gehen beim Virtuosen zusammen « (ebd.). ${ }^{7}$ In seiner Vorführung bestätigt der Virtuose außerdem einen bürgerlichen Arbeitsethos. In der populären Musik ist die Virtuosität nach wie vor ein performativ hervorzubringender Wert, etwa im Gitarrensolo, aber auch im Gesang. Auch beim DJing wird Virtuosität als handwerkliche Professionalität und herausragendes Können mit hohem Schauwert ausgestellt.

\section{Subversion}

Neben diesem konservativ-traditionellen Paradigma der ästhetischen Komplexität, und gelegentlich ihm auch konträr gegenüber, steht auf der progressiven Seite des bürgerlichen Kulturverständnisses die Subversion. Beat und Punk etwa entsprechen kaum den feingeistigen bürgerlichen Ansprüchen an Kunst, aber sie knüpfen an ein Verständnis von Kultur an, das, z.T. in der Nachfolge der Kritischen Theorie, Kunst als Widerspruchs- und Irritationsagenten bestimmt. Einspruch gegen die Verhältnisse zu erheben (vgl. Adorno 2008: 203) - das ist seit den 1960er Jahren ein Paradigma einer avantgardistischen und/oder kritischen Kultur. Dieser Anspruch folgt nicht mehr einem künstlerischen Schönheitsideal, sondern einem aufklärerischen Impuls vor allem im linken Spektrum der Mittelschicht, das über einen gewissen Wohlstand und über (häufig akademische) Bildung verfügt. Diese Teile des bürgerlichen Milieus, die vor allem im Nachklang der 68er an Einfluss gewinnen, haben ein grundsätzliches Misstrauen gegenüber der Kulturindustrie, aber auch gegen das traditionelle bürgerliche Kunstverständnis.

Präsenz und Beliebtheit seit den 1960er Jahren kaum eingebüßt haben, dürfte eine Einschätzung vor allem derjenigen sein, die noch mit ihnen groß geworden sind. Zur Kanonisierung in der Popmusik siehe auch Appen/Doehring (2000) und Appen/Doehring/Rösing (2008).

7 Der Virtuose kann daher als eine Vorläuferfigur des Stars begriffen werden. 
Sie verstehen sich selbst als antibürgerliche Kraft und fordern von der Kultur aufklärerische Impulse, widerständige Kraft und unkonventionelle Originalität. An diese Paradigmen ist eine populäre Kultur, die subkulturell oder sogar gegenkulturell agiert, leicht anschlussfähig. Weil aber nicht alles Populäre subversiv genannt werden kann, weil im Gegenteil die zahlenmäßig populär(st)e Kultur oft auf den ersten Blick vor allem gefallen statt schockieren will, beginnt auch der Begriff >Pop< zu changieren. Beat steckt schließlich in »Love Me Do « der Beatles ebenso wie in den Büchern der Beatliteraten Jack Kerouac, Allen Ginsberg oder William S. Burroughs. Pop kann Roy Lichtensteins Pop Art sein oder einfach Boney M.'s Discomusik. Es beginnt daher eine Binnendifferenzierung in der populären Kultur, die zwischen dem avantgardistisch aufgeladenen Pop-Begriff und der massenmedialen Unterhaltung, zwischen Subkultur und Mainstream unterscheidet.

Zwischen diesen beiden Polen - Komplexität und Subversion - bewegen sich im Wesentlichen die öffentlichen Bewertungen der populären Musik in Wissenschaft, Feuilleton und Publizistik. An drei historischen Momenten der deutschen Popmusikgeschichte lässt sich zeigen, wie diese Dispositionen wirksam werden und ineinander greifen.

\section{Rock'n'Roll und Teenagermusik}

In den 1950er Jahren ist Virtuosität für die populäre Musik noch immer wichtig. Der Schlager schließt ästhetisch an die Vorkriegszeit an, er lebt noch von den Resten seiner Operetten- und Revuefilm-Vergangenheit. Sänger und Sängerinnen wie Rudi Schuricke und Magda Hein führen nicht nur Lieder auf, sondern vor allem ihre Stimmen vor, wenn sie »Wenn bei Capri die rote Sonne im Meer versinkt « oder »Möwe, du fliegst in die Heimat « singen und dabei mit hohen Tönen und Koloraturen ihre künstlerische Qualität darstellen. Auch mehrstimmige Chöre, Orchester und Sologeige zeigen, dass der Schlager der frühen 1950er Jahre auf die Sicherheit eines bürgerlichen Kunstverständnisses baut, das in der Musiktradition des 19. Jahrhunderts verwurzelt ist. Und auch wenn sich der Schlager jener Epoche von diesem Stimmideal, von Chor und Koloratur, bald verabschiedet, bleibt die orchestrale Begleitung noch lange dominant - nicht nur im deutschen Schlager: Erst 1999 wird im Eurovision Song Contest die Orchesterpflicht durch das Halbplayback abgeschafft.

Eine empfindliche Störung dieser an das bürgerliche Kunstverständnis angelehnten Unterhaltungsmusik stellt der US-amerikanische Import des Rock'n'Roll dar. Sowohl die simple Struktur als auch die Lautstärke, der 
schluckauf-Gesang und der dazu gehörende Tanzstil stehen dem bürgerlichen Kunstverständnis entgegen. Es ist nicht nur eine ästhetische, sondern auch eine ideologische Zumutung:

»Unten im Saal, wo 5000 Jugendliche sitzen, wird es warm. Es hält sie nicht mehr auf den Plätzen - die spasmischen Bewegungen des >Bruders, Märtyrers und Gottes< [gemeint ist Elvis Presley, A.d.V.] auf der Bühne pflanzen sich wie eine Welle durch die Bankreihen des Saales fort. Immer zwingender wird der Rhythmus, immer hektischer werden die Bewegungen im Saal. Hier und da springen die Burschen auf, reißen sich die Hemden vom Körper; ihre stumpfen Augen lassen nicht erkennen, ob sie die Umwelt noch wahrnehmen, nur der Über-Rhythmus scheint sie noch voranzutreiben« (Anon 1956: 51f.).

In den Augen der bürgerlichen Erwachsenenkultur erschüttert der Rock'n' Roll buchstäblich Anstand und Moral:

»Wie trunken tanzen einige auf der Straße, von einer sich rhythmisch bewegenden Menge umgeben. Schließlich rotten sie sich zusammen und beantworten Räumungsaufforderungen der Polizei mit lautem Johlen und Pfeifen, so daß es regelmäßig zu den erwünschten Auseinandersetzungen mit der Polizei kommt (Günther Kaiser: Randalierende Jugend [1959], zit. nach Kurme 2008: 188).

Der Untergang des Abendlandes - der Abscheu der bürgerlichen Öffentlichkeit war ein wesentlicher Grund für die Attraktivität des Rock'n' Roll bei den Halbstarken, die gerade nicht aus der Mittelschicht stammen. Kaspar Maase (1992) und andere (Grotum 1994; Kurme 2006) haben gezeigt, dass die Halbstarkencliquen sich vor allem aus Jugendlichen aus dem Arbeitermilieu zusammensetzten, die durch eigene Arbeit das für diesen Lebensstil notwendige Geld verdienten und dadurch eine gewisse Unabhängigkeit hatten. Für sie war die Übernahme US-amerikanischer Kultur und der damit verbunden Verhaltens-Standards Symbol für Freiheit und ein besseres Leben. Mit ihrer Adaption US-amerikanischer Kultur wurden sie zur Avantgarde der Epoche.

Allerdings erweisen sich der deutsche Schlager und die bürgerliche Gesellschaft - nicht zum letzten Mal - als ungeheuer anpassungsfähig. Nach der öffentlichen Hysterie siegen Pragmatismus und ökonomische Interessen. Als Pendant zum Rock'n'Roll entsteht mit dem Teenager-Schlager ein Zwischengenre, das in der Lage ist, den eher aus der Mittelschicht kommenden Jugendlichen ein kulturell adäquates Angebot zu machen. Aus dem rebellischen, mitunter auch gewalttätigen Halbstarken wird ein lässiger Teenager, der einen zivilen Habitus vorlebt und zum Vorreiter einer neuen, fortschritts- und leistungsorientierten Mittelschicht wird. Die dazu konfigurierte 
Musik versöhnt die neue, US-amerikanisch orientierte Mode, die neuen Sounds und Gesangsstile mit den ästhetischen und ideologischen Gesetzen der bürgerlichen Wiederaufbaugesellschaft. Wo die Halbstarken gelangweilt bis grimmig, Zigarette im Mundwinkel, in die Kamera trotzen, lächeln Conny Froboess und Peter Kraus ein strahlendes Gute-Laune-Lächeln.

Eine für diesen Wandel exemplarische Filmszene zeigt eine Gruppe von Teenagern, wie sie sich die Eltern jener Epoche wohl kaum besser wünschen könnten: In dem Conny Froboess-Vehikel Hula Hopp, Conny (1959) bringt eine Gruppe von männlichen und weiblichen Teenagern eine im Tiki-Stil eingerichtete Gaststätte auf Vordermann. Bewaffnet mit Putzlappen, Geschirrtüchern, einem Staubsauger und viel guter Laune, reinigen sie Böden, Gläser, Drachenköpfe. Die Haushaltstätigkeiten sind allerdings lediglich Anlass für ein beschwingtes Lied: »Die Boys und Girls von heute .

»Die Boys und Girls von heute sind junge Leute wie du und ich.

Die Boys und Girls, die lieben

dies schöne Leben wie du und ich.

Doch nichts will man uns schenken

und wenn wir daran denken,

dann haben wir die Zukunft,

die Zukunft in der Hand.

Die Boys und Girls von heute,

die voller Hoffnung durch's Leben geh'n,

Die Boys und Girls von heute,

die brauchen Menschen, die sie versteh'n.«

Die putzende Teenager-Truppe bringt die Verschmelzung von rebellischem Rock' $n$ ' Roll und bürgerlichen Tugenden auf den audiovisuellen Punkt, indem sie - ganz der Wirtschaftswunder-Idee folgend - nicht nur den Staubsauger, sondern vor allem die eigene Zukunft in die Hand nimmt. Es gibt, das zeigt die Szene, inzwischen eine Übereinkunft zwischen den Teenagern und den Erwachsenen, die die Harmonie garantiert: die Erwachsenen - hier in Gestalt der strickenden und mit ihrer Putztruppe hochzufriedenen Wirtin zeigen Verständnis für die Bedürfnisse der Jugend, so lange die sich in die Idee eines aufstrebenden, arbeitenden, letztlich moralisch wie hygienisch sauberen (mittelständischen) Gemeinwesens eingliedern lässt. Die Wirtin lässt die Jugendlichen so putzen, wie es ihnen Spaß macht, dafür ist dann auch alles - auch im metaphorischen Sinne - sauber. Vieles von dem, was noch wenige Jahre zuvor für Aufregung gesorgt hätte, ist, so zeigt die Szene, als akzeptabel ins bürgerliche Repertoire integriert: Die Mädchen tragen 
Hosen und Kurzhaarschnitte, die Jungen Jeans und Karohemd. Tanzen und auf Tische steigen wird so in den Reinigungsvorgang integriert, dass jede noch so vage Erinnerung an Halbstarkenkrawalle abwegig erscheint.

So zahm die Szene angelegt ist - sie ist das Ergebnis eines Wandlungsprozesses. Hier und generell im Teenagerschlager bildet sich ästhetisch ab, was Maase soziologisch und kulturhistorisch beschreibt: Die Übernahme und Integration von Elementen US-amerikanischen Lebensstils führt zu Informalisierungs- und Demokratisierungsprozessen: Die Verhaltensstandards für die ganze Gesellschaft werden mannigfaltiger und differenzierter, gerade weil sie über die neuen Haltungen in einer modifizierten Form Eingang in den Mainstream finden und dadurch jedem zugänglich sind.

»Das Engagement der Kultur- und Konsumgüterindustrie sorgte dafür, daß die weniger starren und strengen Verhaltensstandards nicht in einer Gegenoder Subkultur ghettoisiert wurden, sondern (vor allem über das Normativwerden eines lässigen Typs von Jugendlichkeit) im Lauf der Jahre in die Lebensweise großer Gruppen eingingen« (Maase 1992: 205).

Der Teenager-Schlager integriert diverse musikalische Mittel des Rock'n' Roll ebenso wie Bestandteile des Schlagers. So wird zwar der >Schluckauf-Gesang bei Conny Froboess und Peter Kraus salonfähig. Insgesamt bleibt die gesangliche Phrasierung aber deutsch: Anders als die US-amerikanischen Sänger singen die deutschen Teenager-Stars die Noten ohne Verschleppen oder eigensinniges Vorziehen.

Im Lauf der 1960er und 1970er Jahre schwindet das Unbehagen des Bildungsbürgertums an der populären Kultur. Zum einen ist dies Folge des bereits beschrieben Informalisierungsprozesses, zum anderen ein Ergebnis einer Ausdifferenzierung der populären Musik selbst, die ihren Anspruch auf Wertschätzung u.a. durch mehr Komplexität und höheren intellektuellen Anspruch dokumentiert. Das Konzeptalbum und die Experimente mit den neuen elektronischen Instrumenten gehören dazu, die Ausstellung instrumentaler Fähigkeiten, aber auch die lyrischen Rückgriffe auf die deutsche Romantik durch Bands wie Hölderlin oder Novalis. Gerade in den 1970er Jahren knüpft die deutsche ebenso wie die internationale Popmusik an Ästhetik-Konzepte an, die durch Komplexität und Kunsthaftigkeit überzeugen wollen. 


\section{Punk und Neue Deutsche Welle}

In Konflikt mit diesen elaborierten und distinktionsfähigen Versionen der Popmusik gerät der Punk, der sich nicht mit langwierigem Üben aufhält. Ihm wird mangelnde ästhetische Klasse insbesondere von denen vorgeworfen, die in dieser neuen Pop- bzw. von ihnen eher als Rock- bezeichneten Musik eine kulturelle Heimat gefunden haben. Steve Strange bemerkt 1977 in der Musikzeitschrift Sounds ganz richtig, die musikalische Qualität des Punk gründe sich »eher auf physische Energie und Leidenschaft denn auf technische Befähigung « (Strange 1977: 36) - und er meint es nicht freundlich.

»Der Rock'n' Roll sollte mutig und waghalsig sein, wenn er eine Kraft der Veränderung verkörpern will. Er sollte nicht lässig Gewalttätigkeit und Pessimismus verherrlichen. Er sollte nicht Verbitterung und Gewalt provozieren« (ebd.).

Hier wird rationale Auseinandersetzung statt Leidenschaft gefordert, Veränderung statt Revolution. Es ist eine Haltung, die ganz an das Paradigma vom Einspruch gegen die verhärteten Verhältnisse und damit an eine linke und dennoch im bürgerlichen Mittelstand verortete - Tradition von Kulturkritik anschließen kann und die von der Redaktion zunächst geteilt wird. Punk als »simpler 08/15 Dampfhammer-Rock« (Gillig 1977: 10) könne RockGrößen wie Bob Seeger und Bruce Springsteen, Genesis, Yes oder Supertramp nicht das Wasser reichen. Der Siegeszug des Punk beeindruckt die Sounds-Redaktion nur mäßig, lediglich der freche Umgang der Sex Pistols mit der Plattenindustrie nötigt innen Respekt ab: »jedenfalls haben sie einen Dreh gefunden, den Firmen die Kohle aus dem Kreuz zu leiern, ohne sich groß anzustrengen« (Anon. 1977: 14). Die Dynamik des Punk trifft an anderer Stelle allerdings durchaus auf positives Feedback, wenn nämlich seine subversive Anti-Establishment-Haltung gelobt wird:

»Angesichts dieser Queen, die in ihrer goldenen Prunk-Protz- und Großkotzkutsche da rumfährt und viele, viele Leute in dem Land hängen total in den Seilen, dann finde ich das ganz ordentlich, wenn die Jungs Bambule machen. Das ist jedenfalls besser als die Bay-City-Rollers, mit ihren Wischi-WaschiVerdrängungsliedern« (Udo Lindenberg, zit. nach Lindner 1978: 4).

Der Anspruch auf ästhetische Komplexität und der Anspruch auf Widerstandspotential, treffen im Punk zunächst diametral aufeinander. Die Frage, ob der rohe und ungeschliffene Punk musikalischer Müll ist oder großer 
Widerstand, ist durchaus auch eine Generationenfrage. ${ }^{8}$ Die Begeisterung für das Subversionspotential lässt bei einigen Beobachtern allerdings genau dort nach, wo der Transfer ins Deutsche beginnt.

»Die massenmediale Vermittlung bietet gewissermaßen einen Überbau ohne die dazugehörige Basis. Dies macht, im Falle des Fehlens des inneren sozialen Zusammenhangs, erklärlich, warum exportierte Subkulturen von Arbeiterjugendlichen im Importland z.B. als bloße Stilvariationen zu einem mittelschichtspezischen Phänomen werden können« (Lindner 1981: 190).

Aus einer »authentischen Arbeiter-Subkultur«, die »sichtbarer Ausdruck einer Protesthaltung ist « (ebd.: 191), werde, so der Vorwurf, durch die mediale Vermittlung ein entleertes Konsumgut. Einmal ganz davon abgesehen, dass die Auffassung von Punk als reine Arbeiter-Subkultur eine Legende ist (vgl. Hornberger 2011: 57-69), wird hier eine Haltung deutlich, die seitdem den Indie-Diskurs immer wieder bestimmt: Der Soziologe und Kulturwissenschaftler Lindner, Teil einer intellektuellen und privilegierten MittelschichtKultur, erwartet von der populären Musik Subversion und Revolte. Protest und Widerstand werden in die populäre Musik ausgelagert, um dort als Haltung bei Bedarf verfügbar zu sein, letztlich wie die Bay City Rollers kulturelles Konsumgut, nur für ein anderes Lebensgefühl.

Als sich schließlich aus dem Punk die Neue Deutsche Welle entwickelt, wird der neue Stil nicht nur sozial, sondern auch kulturell von der deutschen Mittelschichtskultur geprägt. Die Szene wird von Musikern beherrscht, die eher als Künstler und Avantgardisten auftreten, die an Konzepte wie Dadaismus und Futurismus anschließen, Hindemith zitieren (vgl. Palais Schaumburg: »Wir bauen eine neue Stadt«, 1981) und die 1920er Jahre neu entdecken?. Sie verweisen vielfach auf die Kulturgeschichte der künstlerischen Avantgarden und beweisen damit vor allem ihre tiefe Verwurzelung im bil-

8 Nach dem Austausch der Chefredaktion zum Juli 1979 wird Sounds von einer Ausgabe zur anderen zur Punk-Verfechterin. Bis dahin zeichnen Jörg Gülden, Jürgen Frey und Teja Schwaner für den eher traditionell ausgerichteten Kurs des Magazins verantwortlich, jetzt werden Frey und Schwaner durch Thomas D. Butler und Diedrich Diederichsen ersetzt, auch Gülden scheidet kurz darauf aus. Im Oktober erscheint in Sounds der erste Teil von Alfred Hilsbergs dreiteiliger NDW-Reportage, mit der das Magazin zu einem der wichtigsten publizistischen Organe der NDW wird.

9 Bands wie die Einstürzenden Neubauten oder Mania D / Malaria! greifen auf Rebellions- und Avantgardebewegungen des frühen 20. Jahrhunderts zurück, sie verweisen insbesondere optisch u.a. auf den Berliner Expressionismus der 1920er Jahre und den Chic linker Arbeitersportverbände. Auch der Schlager der 1920er Jahre wird wiederentdeckt, z.B. durch Coverversionen und Neuinterpretationen von »Bel Ami« (Abwärts, 1980), »Flieger, grüß mir die Sonne« (Extrabreit, 1980) oder »Musik! Musik! Musik!« (The Tanzdiebe, 1982). 
dungsbürgerlichen Milieu. Das kommt bei denen, die sich als »originäre«, nämlich antibürgerliche Punks verstehen, nicht gut an:

»Als erstes tritt der weltaufstandsplan in kraft (oder besser gesagt in krampf). Das warn zwei leute: stimme/synthesizer und gitarre/stimme. Einer war der mann von art attack... wer davon schon was gehört hat, weiß, was von der bühne kam: keine musik!! Geräusche, lärm, texte: abstrakte gedichte aufsagen kurz - der gegensatz von rock 'n' roll, totale mittelstandskultur oder anders: nich mein fall...« (aus dem Fanzine Preiserhöhung, zit. nach Hilsberg 1979: 21).

Abstraktion und Experiment als künstlerische Komplexität - die NDW ist, anders als der Punk, ganz und gar Mittelstandskultur. Mit dieser Art der Mittelstandskultur einher geht ein Exklusivitätsversprechen. Man setzt sich nicht nur gegen den »einfachen « Punk ab, sondern auch gegen den Geschmack der Vorgängergenerationen, die als »BoFs« (boring old farts) bezeichnet werden, sowie gegen den zeitgenössischen Schlager und zeitgenössische Popstile wie etwa Disco. Die Vertreter der frühen NDW sind darum, ebenso wie Journalisten in Sounds und Spex, größtenteils wenig begeistert von der weiteren Entwicklung der NDW, die - in gewisser Ähnlichkeit zur Geschichte des Rock' $n$ 'Roll in der BRD der 1950er Jahre - an das SchlagerGenre anknüpft. Zuerst kann diese Zuwendung noch als ironisch gebrochener Angriff auf dieser Kultur, als taktische Affirmation, wahrgenommen werden, doch als diese Spielart der NDW selbst zum Mainstream wird, ${ }^{10}$ löst sich das Exklusivitätsversprechen auf. Die NDW, entstanden aus der Kultur der gesellschaftlichen Mitte, ist zu sehr Mitte geworden.

\section{HipHop und Deutschrap}

Eine vergleichbare Entwicklung ist auch beim HipHop zu beobachten. In den 1980er Jahren wird die US-amerikanische Kultur in Deutschland zunächst vor allem importiert und adaptiert. Für Jugendliche aus dem bürgerlichen Milieu liefert HipHop wie bereits andere Subkulturen vor ihm eine Möglichkeit zur Abweichung von bürgerlichen Standards und elterlichen Erwartungen. Insbesondere für Migranten ist Rap und Breakdance außerdem ein Angebot, die eigene (oft als ausgegrenzt und strukturell defizitär empfundene) Lebenssituation formuliert zu wissen und selbst zu formulieren.

»Als wir die Filme Wild Style und Beat Street gesehen haben, da war es so, dass sich die Türken und Italiener und so sofort mit den Puerto-Ricanern

10 Im Jahr 1982 wird die ZDF-Hitparade vom NDW-Schlager weitgehend dominiert. 
identifizieren konnten und die Afrodeutschen eben mit den Schwarzen. Das war irgendwie allen sofort klar« (Kutlu, zit. nach Loh/Güngör 2002: 23).

Sie berufen sich auf eine Verwandtschaft mit der schwarzen Importkultur, Rap ist für sie eine sozial aufgeladene kulturelle Praxis, die sich emanzipatorisch gegen eine hegemoniale weiße Mittelstands-Kultur richtet und ethnischen Minderheiten eine Stimme gibt. ${ }^{11}$ Diese Stimme ist zu Beginn englisch, türkisch, italienisch - doch nur selten deutsch.

»Denn Ende der 80er, Anfang der 90er war es nicht cool, auf deutsch zu rappen. Gerade bei vielen Brüdern und Schwestern war die Identifikation mit den Afro-amerikanischen Vorbildern besonders groß - die Gründe dafür liegen auf der Hand: Zu wenig konnte die deutsche Sprache Ausdruck eines positiven Lebensgefühls sein. Dafür hatte sie zu sehr den bitteren Beigeschmack der schmerzhaften Erfahrung des Rassismus. [...] Viele Afros übernahmen daher damals lieber den Habitus und die Musik von ihren US-amerikanischen Vorbildern« (Yakpo 2004).

Nach den Brandanschlägen von Rostock-Lichtenhagen (1992) und Solingen (1993) erhält diese Möglichkeit zur Identitäts- und Positionsbestimmten eine deutlich höhere Relevanz und Brisanz - und eine deutschsprachige Stimme. Mit »Fremd im eigenen Land« legt die multiethnische Heidelberger Band Advanced Chemistry 1992 ein Stück vor, das bis heute paradigmatisch für die Reaktion und Position des migrantischen Rap auf und für eine Auffassung des deutschsprachigen HipHop als Musik der Subversion steht. Der Song wird zu einem Meilenstein, für die bis dahin vor allem migrantisch geprägte HipHop-Szene ohnehin, aber auch für eher bürgerliche Sympathisanten aus PopPresse und Wissenschaft, deren Widerstandsdogma der Song voll entspricht. Interessant im hier verhandelten Kontext ist allerdings, wie dieser Widerstand gesetzt wird. Denn obgleich der Song klar sowohl den rassistischen Gewaltakt als auch die Reaktion darauf kritisiert, ist er nicht in erster Linie ein Song der Distanzierung. Die Rapper erheben im Gegenteil Anspruch darauf, Teil der deutschen Gesellschaft zu sein - sie wollen nicht als »die

11 Für diese identifikatorische Verwandtschaft, die Migranten in Deutschland zwischen dem US-amerikanischem HipHop und dem eigenen Lebensgefühl wahrnehmen, ist eine »echte « soziologische Äquivalenz der Verhältnisse nicht zwingend erforderlich. Auch wenn Ghettos US-amerikanischen Typs in den deutschen Großstädten der 1980er und 1990er Jahre ebenso wenig zu finden sind wie die aus den US-amerikanischen Videobildern bekannten brennenden Ölfässer, reicht für die migrantischen Jugendlichen das Identifikationspotential des US-amerikanischen HipHop aus, um sich den Stil zu eigen zu machen. Insofern läuft die Kritik, diese Art der Positionsübernahme in Stil und Ideologie sei ohne echte soziale Grundlage, ins Leere. Allerdings gilt dies dann auch für den reinen Formentransfer, den der Deutschrap macht (s.u.). 
anderen « wahrgenommen werden, sondern als gleiche, als die »normalen Deutschen «, die sie staatsrechtlich sind: »Ich habe einen grünen Pass mit 'nem goldenen Adler drauf« (»Fremd im eigenen Land«, 1992). Dass diese Demonstration von deutscher Identität ebenfalls eine der Mittelschicht ist, wird - anders als etwa bei Cora E. die sich in »Schlüsselkind « (1996) selbst dort verortet ${ }^{12}-$ im kommunikativen Stil von Stück und Videoclip implizit deutlich. Statt auf Provokation durch Dissen, F-Wörter oder Sexismus setzen die Rapper auf Rationalität, sie zeigen sich als sprachlich versierte, geschichtlich und politisch gut orientierte und vor allem argumentierende Anwälte der eigenen Position.

Während Cora E's biografisches Stück »Schlüsselkind « zwar mit der Feststellung einer Mittelschichtsexistenz beginnt, dann aber eine Geschichte des Scheiterns, des persönlichen und sozialen Absturzes durch elterliche Scheidung, Vernachlässigung und Drogensucht erzählt, in der erst HipHop den rettenden Ausweg darstellt, sind die etwa zeitgleich mit Advanced Chemistry in der Aufmerksamkeit der Öffentlichkeit erscheinenden ${ }^{13}$ Fantastischen Vier Mittelstand durch und durch - und wollen auch gar nichts anderes repräsentieren. Der bei der MTV-Premiere der Band platzierte Satz »Wie are from the Mittelstand « (zit. nach Weber 1999) markiert nicht nur eine Differenz zur US-amerikanischen Herkunftskultur, sondern auch zu einer deutschen HipHop-Adaption, die die Ähnlichkeit der sozialen Situation zu ihrer Voraussetzung bestimmt. Die Fantastischen Vier behaupten an keiner Stelle, aus der Position der oder stellvertretend für die »Subordinated«zu sprechen. »Zum einen hat's für mich den Sinn, zu sagen wer ich bin / zu beschreiben wo ich herkomm', und da ist's halt nicht so schlimm« (»Hip Hop Musik«, 1992), sie sind nie »street«, sondern eher »Reihenhaus«. Ihre Version des HipHop ist reiner Formentransfer (vgl. Burchart 2009: 77ff.) - dafür werden sie stark kritisiert. Insbesondere von der Singleauskopplung »Die da!?!« ist die Szene »entsetzt« (Loh/Güngör 2002: 8).

»Das deutsche Mittelstandsfeeling, das von den Fantastischen Vier in die HipHop-Kultur getragen wurde, war ein klarer Angriff auf das Lebensgefühl der meisten Migranten, die vor allem Rap und Breakdance immer als ihr ureigenes Ding gesehen hatten« (ebd.: 19).

12 »Ich komm' aus einer Mittelschicht / die gibt's in Deutschland sicherlich / Oben war'n wir nicht und unten nicht « (»Schlüsselkind«, 1996).

13 Erstmals treten sie 1991 in Erscheinung auf dem ersten deutschen HipHopSampler Krauts with Attitude mit dem Stück »Jetzt geht's ab und mit ihrem ersten Studioalbum Jetzt geht's ab. 1992 haben sie ihren kommerziellen Durchbruch mit »Die da!?!«, einem Stück, das durch seinen Chart-Erfolg die Vorstellung von Deutschrap maßgeblich prägt. 
Die von Bands wie Advanced Chemistry noch empfundene und vorgetragene soziokulturelle Äquivalenz zu dem Geschehen in US-amerikanischen Großstadt-Ghettos wird von den Fantastischen Vier stilistisch unterlaufen, sie transferieren vor allem einen Musik- und Lebensstil, der ihnen Spaß macht. Damit greifen sie zwar etwas auf, was in der populären Musik schon immer wichtig ist: Spaß. Aber mit >Spaß als Programm sind sie nicht anschlussfähig, nicht für die migrantischen HipHopper, denen sie »zu deutsch« (ebd.: 114) sind, ${ }^{14}$ nicht aus der Perspektive eines sich als politische Stimme verstehenden HipHop-Underground, und auch nicht für einen bürgerlichen Selbstverständigungsdiskurs, der entweder Komplexität oder Subversion verlangt. Für die Subversionsanhänger sind sie zu weiß, zu fun-orientiert, zu kommerziell, zu sehr ZDF-Hitparade. Für die Anhänger von Virtuosität und ästhetischer Komplexität ist zu diesem Zeitpunkt die ganze HipHop-Kultur eine einzige Panzerknacker-Bande, die nur die Musikgeschichte beklaut die Komplexität dieses »Klauens « und die Virtuosität des Flow werden sich erst später als distinktionsfähig erweisen. Vor allem aber sind die Fantastischen Vier viel zu schnell im Mainstream erfolgreich - aus dessen Figurenarsenal sie ja auch ihren Namen haben. ${ }^{15}$ Die Fantastischen Vier werden kaum im Kontext HipHop (als Underground) wahrgenommen, sondern gleich im Kontext Popkultur (als Mainstream). Sie können daher kein Exklusivitätsversprechen geben. Aus diesen Gründen kommt im Fall der Fantastischen Vier der Vorwurf der Ausbeutung ein weiteres Mal zu Ehren: Ihre Adaption einer fremden, als eine Art kultureller Notwehr verstandenen schwarzen

14 »>Die da!?! < ist zwar hier entstanden, aber das war für die Migranten wirklich fremd. Für die Migranten war das zu deutsch « (Volkan, zit. nach Loh/Güngör 2002: 114). An der »Eindeutschung « des Rap stoßen sich die in der HipHop-Szene beheimateten Migranten ganz besonders. Dies prägt auch Jahre später noch den Ton des Buchs Fear of a Kanak Planet (Loh/Güngör 2002). Allerdings übersehen die Autoren des Buchs, dass die Eroberung der deutschen Sprache für Popmusik (durch die NDW) erst zehn Jahre her und damit vergleichsweise jung ist. Darum wird etwa auch die Aufschrift auf dem Sampler Krauts with Attitude (1991) - »Es ist Zeit, dem Selbstbewusstsein der Engländer oder der Amerikaner irgendwas entgegenzusetzen « - ahistorisch und damit falsch interpretiert. Hier geht es nicht patriotisch um eine »Werkschau deutschen Kulturguts« (ebd.: 42), sondern um die Fortführung bzw. Verteidigung einer mühsam und spät errungenen - und zu diesem Zeitpunkt noch keineswegs selbstverständlichen eigenen Sprechweise in einer weitgehend angloamerikanisch beherrschten Popmusik. Zur Bedeutung der Deutschsprachigkeit in der NDW siehe Hornberger (2011).

15 Die Fantastischen Vier sind ein Superhelden-Team des amerikanischen ComicVerlags Marvel, die Reihe startete 1961. Anders als heute gelten Comics in den 1950er und 1960er Jahren nicht als kunstvolle bildliche Erzählungen, sondern als anrüchiger Teil populärer Kultur. 
Ghettokultur könne nur als kommerzielle und kulturelle Ausbeutung abgelehnt werden.

\section{Vom Drama einer Kultur}

Die Fantastischen Vier haben sich explizit wie kaum eine andere HipHopBand zu ihren Wurzeln in der deutschen Mittelschicht bekannt. Bei diesem Bekenntnis bleiben sie auch, als es kaum mehr relevant ist, weil die Diversifizierung des HipHop in Deutschland den Streit über die Herkunft verblassen lässt und sich die Band im Lauf der Jahre breite Anerkennung erwirbt. Die Verwendung des (eingangs zitierten) Pyramiden-Show-Textes, den sie auf einer alten Schallplatte finden, passt insofern in die Strategie, ebenso die Begründung, die Smudo dafür gibt:

»Das Intro stammt von einer Hörspiel-Platte aus dem Jahr 1961, so 'ne Art musikalisches Museum über Ägypten, Pharaonen und so. Wir fanden das ziemlich passend, weil wir in $>\mathrm{MfG}<$ ja das Drama dieser Kultur von hier und heute erzählen. Wir rappen die ganze Zeit Abkürzungen, eigentlich sagen wir also nichts, und trotzdem sagen wir alles, weil die Abkürzungen für Werte, Moralvorstellungen, Institutionen, ach, einfach für alles mögliche stehen« (Smudo, zit. nach Weber 1999).

Tatsächlich ergibt die gereimte Reihung von Abkürzungen eine genau beobachtete Diagnose jüngster deutscher (Kultur-)Geschichte. Natürlich sagen die Fantastischen Vier hier keineswegs nichts, denn die Zusammenstellungen sind nicht nur dem Reim, sondern einer inhaltlichen Logik geschuldet:

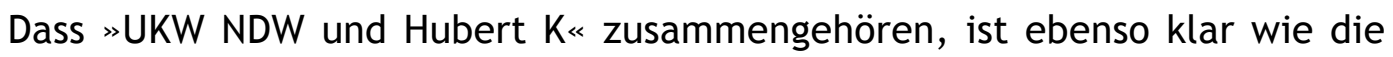
Verbindung von »EDV IBM und WWW . Das erste skizziert die NDW - in ihrer Hitphase -, das zweite eine wesentliche technologische Entwicklung. Bei »TUI UVA und UVB« hat man die Urlaubsstrände vor Augen, bei »BSE HIV und DRK« die diversen Gesundheitsskandale. Die Abkürzungen ergeben ein Panorama zeitgenössischer deutscher Kultur - und sie sind nicht nur harmloses Abkürzungsmemory, sondern spitzen allein durch die Zusammenstellung auch Sachverhalte zu: Die Reihe $\gg \mathrm{H}+\mathrm{M}$ BSB und $\mathrm{FDH}$ « etwa verbindet den Modekonzern, Wirtschaftsstatistik und Körperoptimierung durch Diäten, »FAZ $B W L$ und FDP « weist ein bedeutendes bundesdeutsches Presseorgan als politisch klar positioniert aus, und die Reihung »KMH ICE und Eschede« erinnert an die folgenschwere Zugkatastrophe. Dies alles ist natürlich nicht zufällig, die Band ist sich, wie das Zitat oben zeigt, klar bewusst, dass die von ihnen verwendeten Abkürzungen eine moralische Landkarte abbilden und dass ihre Anordnung ein hochcodierter und verdichteter Rätselapparat ist, der ad- 
äquate Dekodierung verlangt. Damit fordern sie einen informierten und wachen Hörer. Nebenbei gehört erschließen sich kaum die inhaltlichen Zusammenhänge und damit einhergehenden feinen Spitzen.

Weil der Text originell, präzise und fordernd ist, kann er an den Anspruch von Komplexität anschließen. Zwar sind nach wie vor die Subversionspotentiale bei den Fantastischen Vier nur wenig ausgeprägt, aber dafür zeigen sich die Fantastischen Vier im Song und im Video als selbstreflexive Grenzgänger zwischen US-amerikanischer und deutscher Kultur, zwischen Feingeist und Kulturunternehmer. Das Kostüm, viermal gleiches Hemd und Krawatte, und eine synchronisierte Minimalchoreografie symbolisieren eine mittelständische Angestellten-Kultur, Individualität ist hier zweitrangig. Während der ersten Strophe sieht man die Rapper an einem Riesenkopierer, dessen Bilder die Abkürzungen ebenso erhellen wie bereichern. Die wie Dias zu den Abkürzungen eingeblendeten Bilder sind die zweite Ebene einer ästhetisch anspruchsvollen Verweisstruktur, der Riesenkopierer kann zugleich als Anspielung auf die Vorwürfe der bloßen Stil-Kopie gelesen werden. Im zweiten Teil des Videos wird das Setting US-amerikanischer (und endet auf einer Straße mit Palmen, die einen Sonnenstaat wie Kalifornien oder Florida markiert). Das Video visualisiert so das Grenzgängertum der Bands zwischen USA und BRD, zwischen dem Herkunftsland des HipHop und ihrem eigenen Ort. Eine ebenfalls uniformierte Putztruppe aus jungen Frauen verwandelt sich in Cheerleader, deren perfekte Choreografie von den weniger perfekten Einlagen der Fantastischen Vier ironisch konterkariert wird. Der Refrain stellt mit einer weiteren Abkürzung, MfG, eine Verbindung her zwischen einer höflichen Büro-Kommunikation und der Lässigkeit der damals noch neuen - Mail-Kommunikation.

»MfG mit freundlichen Grüßen

die Welt liegt uns zu Füßen denn wir stehen drauf

wir gehen drauf für ein Leben voller Schall und Rauch

bevor wir fallen fallen wir lieber auf«

(Die Fantastischen Vier: »MfG«, 1999).

Der Refrain beschreibt die Position der Band in dem Diskurs um HipHop als politisches Statement oder Lebensstil und damit ein Dilemma, das alle deutschen Popbands betrifft, die die Balance zwischen dem Streben nach Erfolg und dem Vermeiden des Mainstream-Vorwurfs suchen. Im nunmehr fünften Studioalbum heißt das Fazit der Fantastischen Vier: Sie stehen auf den Erfolg und genau darum liegt ihnen die Welt zu Füßen. Sie haben sich immer dafür entschieden, ihren eigenen Mittelstands-Status - im >Ländle`zählen 
Leistung und Erfolg - nicht zu verschleiern, sondern selbstbewusst, aber auch selbstreflexiv auszuspielen, bis hin zum eingangs zitierten Song-Intro.

Es entsteht durchaus eine intellektuelle und moralische Schieflage, wenn aus der Mittelschicht stammende (und von ihr in der Regel auch lebende) Akademiker und Journalisten, immerhin einer gesellschaftlichen Gruppe angehörend, die gesellschaftlich und ökonomisch begünstigt ist, für ihr populäres Vergnügen den Ausdruck derer nutzen, die weniger profitieren. Zugespitzt formuliert: Für die lebensstilistische Formulierung des eigenen Unbehagens wird die Stimme jener in Anspruch genommen, deren prekärer Status eine Nebenfolge der eigenen privilegierten Situation ist. ${ }^{16}$ Die Erwartung von Protest seitens der Subkulturen ist darum häufig gekoppelt an die Erwartung, dass der Status des Draußen-Seins aufrechterhalten wird; dies gilt jedenfalls dann, wenn kommerzieller Erfolg als Ausverkauf und Anbiederung denunziert wird. Die heimliche Sehnsucht der Mittelstands-Vertreter nach dem >Ehrlichen< und >Subversiven< der >Subordinated < ist aber nicht nur sozialromantisch naiv, sie instrumentalisiert und stabilisiert den sozialen Gap und sie verstellt den Blick auf wesentliche Prozesse kultureller Entwicklung. Denn kulturelle Produkte werden immer abhängig von ihrem kulturellen, gesellschaftlichen und historischen Kontext hergestellt und rezipiert. Darum hinterlässt ihr Transfer Lücken, er verursacht Teil- und Missverständnisse. Die Übertragung von kulturellen Artefakten und Stilen von einem sozialen oder kulturellen Kontext in den anderen kann niemals voll-

16 Anders verhält es sich mit der Hamburger Schule, wo Künstler und Kritiker etwa dem gleichen sozialen und kulturellen Milieu angehören. Bürgerliche Pop-Künstler singen von den Fragen ihrer bürgerlichen Jugend, wofür sie von der Presse gefeiert werden, die in der intellektuellen Sperrigkeit der Lieder Komplexität und Widerstand zugleich vereint findet. Die Bands der Hamburger Schule beziehen die Reflektion ihrer bürgerlichen Herkunft Situation mitunter ironisch mit ein. Anders als bei den Fantastischen Vier gilt dies hier aber nicht als Verrat an der subversiven Grundidee, sondern im Gegenteil als selbstreflexives Spiel mit dem eigenen gesellschaftlichen Status.

»Warst du nicht fett und rosig.

Warst du nicht glücklich,

bis auf die Beschwerlichkeiten.

[...]

Hast du denn niemals richtig rebelliert.

Kannst du nicht richtig laufen,

oder was lief schief.

Und sitzt die Wunde tief in deinem Innern.

Kannst du dich nicht erinnern.

Bist du nicht immer noch Gott weiß wie privilegiert.

Was hat dich bloß so ruiniert.

Was hat dich bloß so ruiniert «

(Die Sterne: »Was hat dich bloß so ruiniert«, 1996). 
ständig und deckungsgleich sein, weil sich durch einen neuen Kontext und neue Protagonisten auch neue Bedeutungsspielräume ergeben. Und je pointierter Produkte auf die nationalen Gegebenheiten ihrer Entstehungsorte zugeschnitten sind, desto höher sind die beim Import entstehenden Reibungsverluste und Bedeutungsverschiebungen.

»Weltweit verbreitete Kulturprodukte nivellieren Unterschiede, verschärfen sie aber auch. Michael Jackson bedeutet nicht überall dasselbe. Das gilt noch viel mehr bei den zugespitzten Semantiken von politisch codierten Kulturprodukten« (Diederichsen 1993: 166).

Egal, was und von wem Kultur importiert und angeeignet wird - in der deutschen Popmusik, selbst da, wo sie an die englische oder US-amerikanische deutliche Anleihen nimmt, scheint fast immer eine bürgerliche Grundierung durch. Sowohl die ästhetischen Verfahrensweisen als auch die Bewertungskategorien in der deutschen Popmusik stammen überwiegend aus dem Repertoire des bürgerlichen Salons, der künstlerischen Avantgarde oder der linken Intelligenz und damit aus verschiedenen Facetten bürgerlicher Kulturtradition. Dies gilt übrigens für Ost und West gleichermaßen, auch wenn es sehr unterschiedliche Ausprägungen erfährt. Die bürgerliche Grundierung lässt sich in den sorgfältig gezupften Gitarrenbegleitungen der Liedermacher genauso finden wie in den lyrischen Texten des Ost-Rocks, in den hoch kodierten Wortspielen des Deutsch-Raps ebenso wie in den Soundexperimenten von Can.

Die durch den Kulturtransfer entstehenden Mixturen aus fremdem und eigenem Material müssen nicht zwangsläufig als Verfälschungen und Verlust betrachtet werden. Sie zeigen auf der Habenseite auch die ungeheure Wandlungs- und Entwicklungsfähigkeit von Kultur. Der Rock'n' Roll verändert und modernisiert den Schlager, NDW und deutscher HipHop bilden sich als eigenständige und vielfältige Stile aus. Sobald diese neuen modifizierten Formen im Mainstream angekommen sind, zeigen sie aber nicht nur ihre Kommerzialität, sondern auch, dass ihre Ästhetik und ihre Programmatik mehrheitsfähig geworden sind, sich also die Mitte der Kultur bereits verändert hat. Wenn populäre Kultur überhaupt Gesellschaft verändern soll, geht der Weg fast zwangsläufig über den Mainstream, und der ist - in allen möglichen Variationen - bürgerlich. 


\section{Literatur}

Ackerknecht, Erwin (2007). »Der Kitsch als kultureller Übergangswert« [1934]. In: Kitsch. Texte und Theorien. Hg. v. Ute Dettmar und Thomas Küpper. Leipzig: Reclam, S. 137-155.

Adorno, Theodor W. (2008). »Résümé über Kulturindustrie« [1967]. In: Kursbuch Medienkultur. Die maßgeblichen Theorien von Brecht bis Baudrillard. Hg. v. Claus Pias, Joseph Vogl, Lorenz Engell, Oliver Fahle und Britta Neitzel. München: DVA, S. 202-208.

Anon. (1956). »Rock'n'Roll. Der Über-Rhythmus.«In: Der Spiegel, H. 39, 26. September, S. 51-54; http://www.spiegel.de/spiegel/print/d-31882324.html (Zugriff 9.7.2014).

Anon. (1977). »Der goldene Schuß.«In: Sounds, H. 5, S. $14 \mathrm{f}$.

Appen, Ralf von / Doehring, André (2000). "Kanonisierung in der Pop-/Rockmusik oder: Warum Sgt. Pepper? Zur ästhetischen Beurteilung von Pop-/Rock-LPs in 100er Listen.« In: Populäre Musik im kulturwissenschaftlichen Diskurs. Hg. v. Helmut Rösing und Thomas Phleps (= Beiträge zur Popularmusikforschung 25/26). Karben: Coda, S. 229-249.

Appen, Ralf von / Doehring, André / Rösing, Helmut (2008). »Kanonbildungen in der populären Musik. Pop zwischen Geschichtslosigkeit und Historismus. " In: No Time for Losers. Charts, Listen und andere Kanonisierungen in der populären Musik. Hg. v. Thomas Phleps und Dietrich Helms (= Beiträge zur Popularmusikforschung 36). Bielefeld: transcript, S. 25-49.

Bennett, Tony (2010). »Der bürgerliche Blick. Das Museum und die Organisation des Sehens. «In: Die Ausstellung. Politik eines Rituals. Hg. v. Dorothea Hantelmann und Carolin Meister. Zürich, Berlin: diaphanes, S. 47-77.

Diederichsen, Diedrich (1993). Freiheit macht arm. Das Leben nach Rock'n'Roll 1990-93. Köln: Kiepenheuer \& Witsch.

Fiske, John (1989). Reading the Popular. Boston: Unwin Hyman.

Gillig, Manfred (1977). »Viel Stunk um Punk. In: Sounds, H. 2, S. 12.

Grotum, Thomas (1994). Halbstarke. Zur Geschichte einer Jugendkultur in den 50er Jahren. Frankfurt/M.: Campus.

Hebdige, Dick (1979). Subculture. The Meaning of Style. London: Methuen.

Hecken, Thomas (1997). »Vorwort.«In: Der Reiz des Trivialen. Hg. v. dems. Opladen: Westdeutscher Verlag, S. 7-12.

Hecken, Thomas (2007). Die Theorien der Populärkultur. Dreißig Positionen von Schiller bis zu den Cultural Studies. Bielefeld: transcript.

Hilsberg, Alfred (1979). »Neue deutsche Welle. Aus grauer Städte Mauern.« In: Sounds, H. 10, S. 20-25.

Hornberger, Barbara (2011). Geschichte wird gemacht. Die Neue Deutsche Welle. Eine Epoche deutscher Popmusik (= Film - Medium - Diskurs 30). Würzburg: Königshausen \& Neumann.

Hügel, Hans-Otto (2003). »Virtuose.«In: Handbuch Populäre Kultur. Hg. v. dems. Stuttgart, Weimar: Metzler, S. 491-496.

Jürgens, Christian (1999) »We are Mittelstand. Die Fantastischen Vier melden sich mit Buch und CD zurück. «In: Zeit Online, 29. April; http://www.zeit.de/1999/ 18/199918.fantastischen_.xml (Zugriff: 9.7.2014).

Kurme, Sebastian (2006). Jugendprotest in den 1950er Jahren in Deutschland und den USA. Frankfurt/M.: Campus. 
Lindner, Rolf (Hg.) (1978). Punk Rock. Frankfurt/M.: Verlag Freie Gesellschaft.

Lindner, Rolf (1981). »Jugendkultur und Subkultur als soziologische Konzepte.« Nachwort zu: Mike Brake: Soziologie der jugendlichen Subkulturen. Eine Einführung. Hg. v. Rolf Lindner. Frankfurt/M.: Campus, S. 172-193.

Loh, Hannes / Güngör, Murat (2002). Fear of a Kanak Planet. HipHop zwischen Weltkultur und Nazi-Rap. Höfen: Hannibal.

Maase, Kaspar (1992). BRAVO Amerika: Erkundigungen zur Jugendkultur der Bundesrepublik in den fünfziger Jahren. Hamburg: Junius.

Strange, Steve (1977). »Punk Rock. Die Rückkehr der Rotznase.«In: Sounds, H. 1, S. 34-37.

Tenbruck, Friedrich (1986). »Bürgerliche Kultur.«In: Kölner Zeitschrift für Soziologie und Sozialpsychologie. Sonderheft Nr. 27: Kultur und Gesellschaft. Opladen: Westdeutscher Verlag, S. 263-285.

Wagner, Peter (1999). Pop 2000. 50 Jahre Popmusik und Jugendkultur in Deutschland. Das Begleitbuch zur 12-teiligen Sendereihe des WDR in Co-Produktion mit den Dritten Programmen der ARD. Hamburg: Ideal.

Weber, Martin (1999). „Die Fantastischen Vier. Absolute Gewinner."In: Musikexpress, H. 5; http://www.musikexpress.de/das-archiv/article283320/die-fanta stischen-vier.html (Zugriff: 2.7.2014).

Willis, Paul E. (1978). Profane Culture. London: Routledge.

Winckelmann, Johann Joachim (1995). Gedanken über die Nachahmung der griechischen Werke in der Malerei und der Bildhauerkunst. Stuttgart: Reclam [orig. 1755].

Yakpo, Kofi (2004). »>Denn ich bin kein Einzelfall, sondern einer von vielen<. Afrodeutsche Rapkünstler in der Hip-Hop-Gründerzeit. «In: Afrikanische Diaspora in Deutschland. Dossier der Bundeszentrale für Politische Bildung; http://www. bpb.de/gesellschaft/migration/afrikanische-diaspora/59580/afro-deutsche-rapk uenstler?p=all (Zugriff: 2.7.2014).

\section{Diskographie}

Abwärts (1980). »Bel Ami«. Auf: AmokKoma. LP, Zickzack ZZ 10.

Advanced Chemistry (1992). »Fremd im eigenen Land.«Maxi-CD, MZEE Records AC $01 \mathrm{CD} 92$.

Cora E. (1996). »Schlüsselkind. «Maxi-CD, MZEE 030 CD.

Die Fantastischen Vier (1991). Jetzt geht's ab. CD, Columbia COL 4688622.

Die Fantastischen Vier (1992). »Hip Hop Musik.« Auf: 4 gewinnt!. CD, Columbia COL 4722632.

Die Fantastischen Vier (1999). 4:99. CD, Columbia 4942386.

Die Sterne (1997). »Was hat Dich bloß so ruiniert?« Maxi-CD, Epic EPC 6630822.

Extrabreit (1980). »Flieger, grüß mir die Sonne. « Auf: Ihre größten Erfolge. LP, Reflektor Z 0060.348.

Palais Schaumburg (1981). »Wir bauen eine neue Stadt.« Auf: Palais Schaumburg. LP, Phonogram 6435139.

The Tanzdiebe (1982). »Musik! Musik! Musik!« Auf: Various Artists - Teutonik Disaster. CD, GOMMA 032.

Various Artists (1991). Krauts with Attitude. German HipHop Vol. 1. CD, Boombastic Records 262039. 


\title{
Filmographie
}

Hula Hopp, Conny (1959). Regie: Heinz Paul. DVD: KNM Home Entertainment, 2006. The Spy Who Loved Me (1977). Regie: Lewis Gilbert. DVD: Twentieth Century Fox Home Entertainment, 2007.

\begin{abstract}
In German tradition popular culture was and is either dismissed as a degenerated form of high culture or valued as culture of restistance of the subordinated people. The first concept stands in the tradition of $19^{\text {th }}$ Century German middle class, the second has has its origins in by the British and American Cultural Studies since the 1960s. The first aims at artistic complexity, the second at resistance and subversion. As diverse as they might appear at first sight, both concepts can be related either to the salons bourgeois or the artistic avantgarde or the cultural criticism of the left; and both miss mainstream culture, usually neither subversive nor complex but popular.

This essay focuses on the relationship that German pop musicians as well as the German pop critics and scholars (usually belonging to the middle class themselves) have to this middle class tradition that is both: "Leitkultur « and foe. It explores how such bourgeois traditions of valuation persist in German discourse using the examples of three historical moments of German pop history, when imported American or British pop music was transferred into a German mainstream style: Rock'n' Roll turning into teenage Schlager, Punk into NDW, American HipHop into German Rap.
\end{abstract}

